\title{
FETUS FUTURES
}

\author{
L. Joseph Butterfield, M.D.
}

It has been more than a century since the French obstetricians extended intensive care of the mother to the benefit of the newborn infant. Led by the Parisians, Etienne Stephane Tarnier, who invented the first closed incubator for the premature infant, and Pierre Constant Budin, his protege who extended the immediate care of the newborn into support of the mother in the care of her newborn infant, the French obstetricians established the foundation of what we know today as pediatrics and neonatology.

Twenty five years ago the past president of the American College of Obstetricians and Gynecologists, Sprague Gardiner, accepted the point leadership in a revolutionary concept called Regional Perinatal Care. This simple statement of a mission called for the recognition of the high-risk mother in time to move her to a regional perinatal center ... where appropriate ... for delivery/or to be prepared to move the sick baby of a low- or high-risk mother to a regional neonatal center for care.

The late William Tooley, who was the first chairman of the AAP Section on Perinatal Pediatrics, and the late L. Stanley James, who with his colleague, Virginia Apgar, described the perimeters of perinatal asphyxia, lent their clinical skills and their academic reputations to the concept of regional perinatal care. Apgar, though whose scoring system's "eyes" every baby in the civilized world is first seen, herself was a key player in recruiting the support of the March of Dimes/Birth Defects Foundation in financing the National Committee on Perinatal Health, which crafted "Toward Improving the Outcome of Pregnancy." That landmark monograph was published in 1976 in an unusual consortium of providers, the AAP, the AAFP, the ACOG, and the AMA.
Toward Improving the Outcome of Pregnancy $(T I O P)$ provided a blueprint for the organization of a system of regional perinatal care which, in many variations on the theme, has been adopted worldwide over the last quarter century.

More and more premature and sick infants have been transported to regional neonatal centers and fewer and fewer high-risk mothers have been left to deliver in the less than intensive care capable obstetric units since TIOP rose into the clinical heavens of American medicine.

The net effect, coupled with advances in the technology of perinatal care such as prenatal steroids and postnatal surfactant therapy, has been an improvement in the outcome of pregnancy that Dr. Sprague Gardiner called for back in 1971!

At one of the classical dinner parties of the AMA Maternal and Child Care Committee at the Drake Hotel in Chicago in the early 1960s, a British Obstetrician/Gynecologist to whom the Queen of England trusted her care, gave these after dinner remarks, ". . . one of our esteemed medical historians, pointed out that the future of a nation depends, in major part, on the care that it extended women at the time of childbirth."

In their analysis of neonatal morbidity and mortality in maternal (in utero) and neonatal transports at 24-34 weeks' gestation, Dr. Philip Shlossman and his colleagues (see pp 449-456, this issue) have given a solid scientific basis for the challenge of Dr. Sprague Gardiner and his AMA Committee on Maternal and Child Care over a quarter of a century ago when they called for their contemporaries in obstetrics, family practice, and pediatrics to adopt regional perinatal care as the standard of care of the pregnant woman and her child in the United States.

University of Colorado School of Medicine/The Children's Hospital, Denver, Colorado

Reprint requests: Dr. Butterfield, Pediatrics Department, University of Colorado School of Medicine/The Children's Hospital, Denver, CO 80210 